\title{
Application of the continuous no-reset switching iterative learning control on a novel optical scanning system
}

\author{
Jia-Yush Yen ${ }^{\mathrm{a}, *}$, Yea-Chin Yeh ${ }^{\mathrm{a}}$, Yung-Hao Peng ${ }^{\mathrm{a}}$, Jyh-Fa Lee ${ }^{\mathrm{b}}$ \\ a Department of Mechanical Engineering, National Taiwan University, No. 1, Sec. 4, Roosevelt Road, Taipei 10617, Taiwan, ROC \\ ${ }^{\mathrm{b}}$ Materials and Electro-Optics Research Division, Electro-Optics Section, Chung-Shan Institute of Science and Technology, Lung-Tan, Tao-Yuan 32599, Taiwan, ROC
}

\section{A R T I C L E I N F O}

\section{Article history:}

Received 31 August 2007

Accepted 23 June 2008

Available online $\mathrm{xxxx}$

\section{Keywords:}

Iterative learning control

Piezo-electric stage

Optical scanning system

\begin{abstract}
A B S T R A C T
Modern optical scanning systems often use the advanced servo system to enhance the scanning accuracy and to increase the field of view. This paper presents a continuous no-reset switching iterative learning control algorithm for a novel optical scanning system to achieve the requirements of both fast response and wide field of view. In addition, the proposed method overcomes the non-converging reset error problem experienced by most conventional iterative learning control algorithms.
\end{abstract}

() 2008 Elsevier Ltd. All rights reserved.

\section{Introduction}

Modern optical scanning systems emphasize on the scanning range, scanning speed and the scanning resolution. A smart mechanism with very high servo performance is most suitable for these uses; therefore, it has become very popular in the optical scanning applications. Lately, a new step-and-scan path in Fig. 1 has been from based on the knowledge of the previous operations of the same task [2]. Therefore, this study has also chosen the ILC to achieve the high performance servo requirements.

It is noted that the ILC algorithm, assumes that the initial state of the plant is equal to that of the desired trajectory so that perfect tracking can be achieved. While it is very difficult to assign the initial states in practice, many studies had discussed the design of robust ILC input against initial state errors [3]. There are different structures proposed for fixed initial state error or bounded variable initial state error [4-7], even so all the articles have to constrain the initial states to within a region. In 1996, Sison and Chong brought forth an interesting concept of no-rest iterative learning control (NRILC) [8] to convert the iterative procedure in the linear time invariant single-input-single-output (SISO) system into an equivalent system for stability analysis. The stabilizing control in their paper is not structured for practical application and they did not discuss the issue of initial state error nor offer any simulation or experiment results.

This paper first extends the conventional ILC setting to the multi-input-multi-output (MIMO) situation for the application to

\footnotetext{
* Corresponding author. Tel.: +886233662688

E-mail address: jyen@ntu.edu.tw (J.-Y. Yen).
}

a novel high-speed optical scanning mechanism system. A continuous iterative learning control (CILC) algorithm is then proposed to suppress the initial state error that often diverges upon ILC. The system architecture and identification results are depicted in Section 2. The traditional ILC control criteria are briefly described in Section 3. This section also proposes the use of the traditional ILC control criterion to simulate the NRILC response, termed the continuous iterative learning control (CILC) to distinguish the NRILC in [8]. The original CILC produces poor system responses because it violates the assumptions for the conventional ILC. Section 3.2.2 then presents a modified CILC strategy noticed in several optical systems, particularly for the long distance and large field scanning systems. There are $4 n$ steps in the step-and-scan path. This scanning path is usually arranged for an array of charge coupled devices (CCD) or complementary metal oxide semiconductor (CMOS) detectors. The signal from a single CCD or CMOS detector image displays only a small portion of the front view, and the full view is reconstructed by stitching the images together. To reconstruct a truthful front image, the stepping motion requires not only a precise position control but also a mechanism that can provide a large acceleration upon request. The conventional high torque actuators and its driving mechanisms always take up huge spaces. The proposed novel optical scanning system [1], on the other hand, uses piezo-electric transducers (PZT) with flexure joints and is very compact in size. From the servo point of view, the step-and-scan motion requires sufficient control sampling points during each step; however, there is a hardware limit on the number of usable sampling points. It is both unwise and uneconomic to change the hardware to accommodate for the growing step number $n$. Thus, making use of the previous control information for self control 


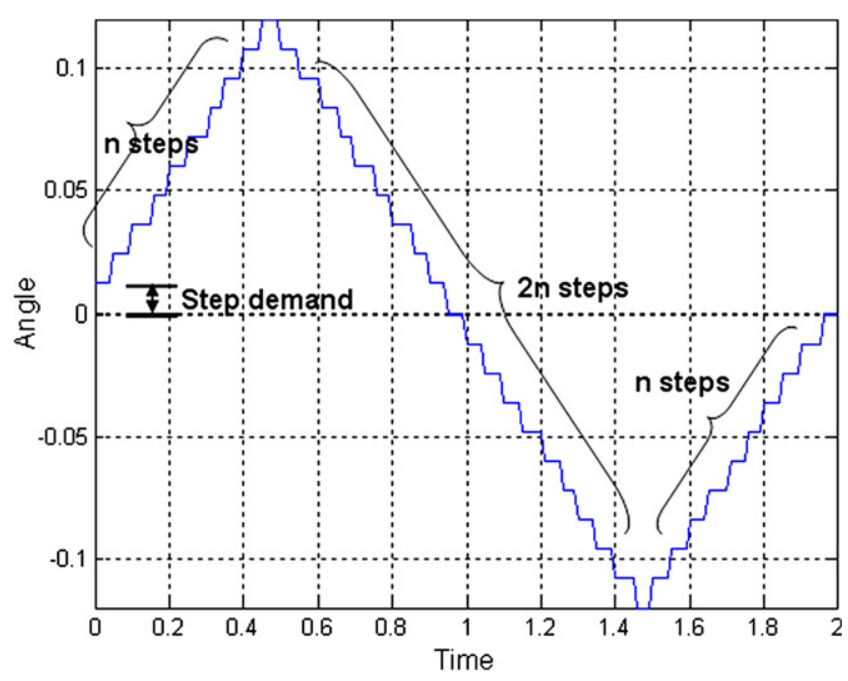

Fig. 1. The step-and-scan path.

tuning would be the best way to suppress the tracking error. There are mainly two types of learning control that serves the purpose: the repetitive control (RC) and the iterative learning control (ILC).

The RC design eliminates the periodic system disturbances and periodic steady state error, but it along does not improve the transient tracking performance. There are many modifications to the RC methods to enhance the steady state error performance, but they all suffer from the compromises between achievable phase compensation and system instability. The ILC is an effective method for reducing tracking errors from repeated operations. The ILC is robust against model uncertainties; it requires no detailed prior knowledge about the system; it modifies an unsatisfactory control input overcome the reset assumption. The modified CILC strategy is then extended to the MIMO case. The simulation outcomes in Section 3.2.3 verify that the modified CILC strategy is efficient. The section then discusses the non-zero initial error problem for some various optical servo systems. Section 4 displays the experimental results. Both simulation and experimental results of this application confirm that the proposed method can effectively suppress the iterative learning error and achieve the desired performance.

\section{The novel optical mirror scanning system}

The optical scanning system uses a quick scanning mirror to reflect pieces of images from different part of the field-of-view onto the CCD array. To truthfully represent the original image, the scanning mirror must be fast to scan the entire field-of-view and precise to determine the position of each pixel. It is thus import to design a high performance servo mechanism for the scanning mirror. Fig. 2 shows the architecture of the novel mirror scanning system; two multilayer piezo actuators "A", "B" are connected to the flexure joints "a", "b" of the scanning stage to drive the mirror. The flexure joint helps avoid mechanical problems such as backlash, friction and lubrication. The multilayer PZT helps attain high output displacements and high scanning speed. Applying different driving voltages to " $\mathrm{A}$ " and " $\mathrm{B}$ " induce an elongation difference which twists the scanning mirror through the flexure joints to produce the desired mirror scanning angle. Two linear variable differential transformers (LVDT), "C" and "D", measure the actuator elongations for the control feedback. The control system is illustrated in Fig. 3.

The proposed novel optical scanning system is an MIMO scanning system. The experiment used an Agilent 35670A dynamic

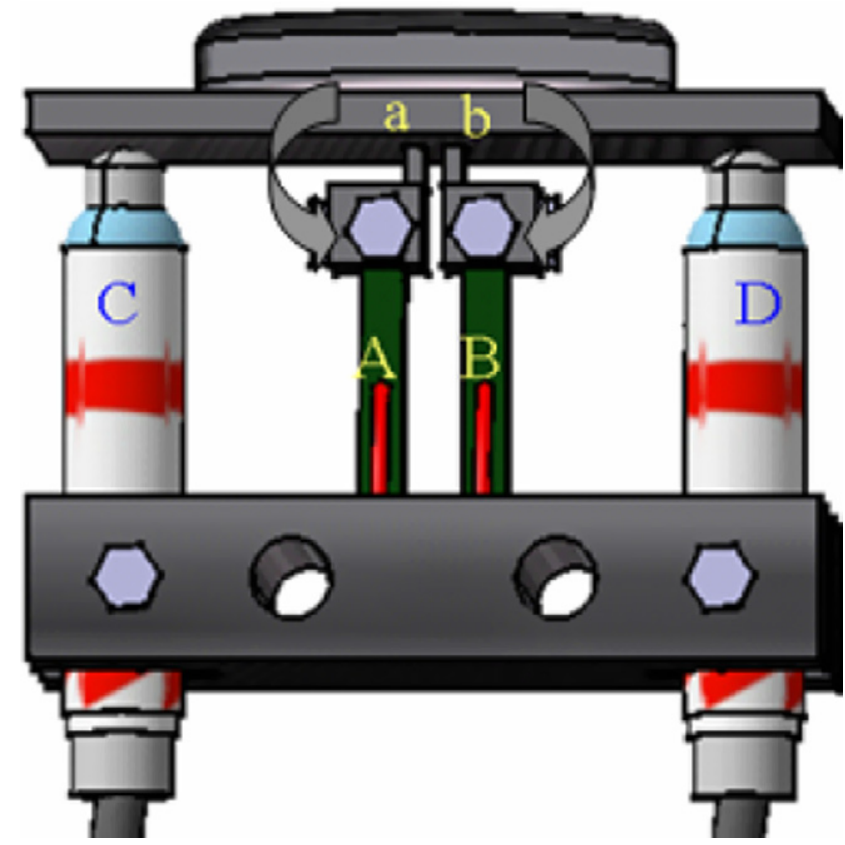

Fig. 2. Mechanism diagram.

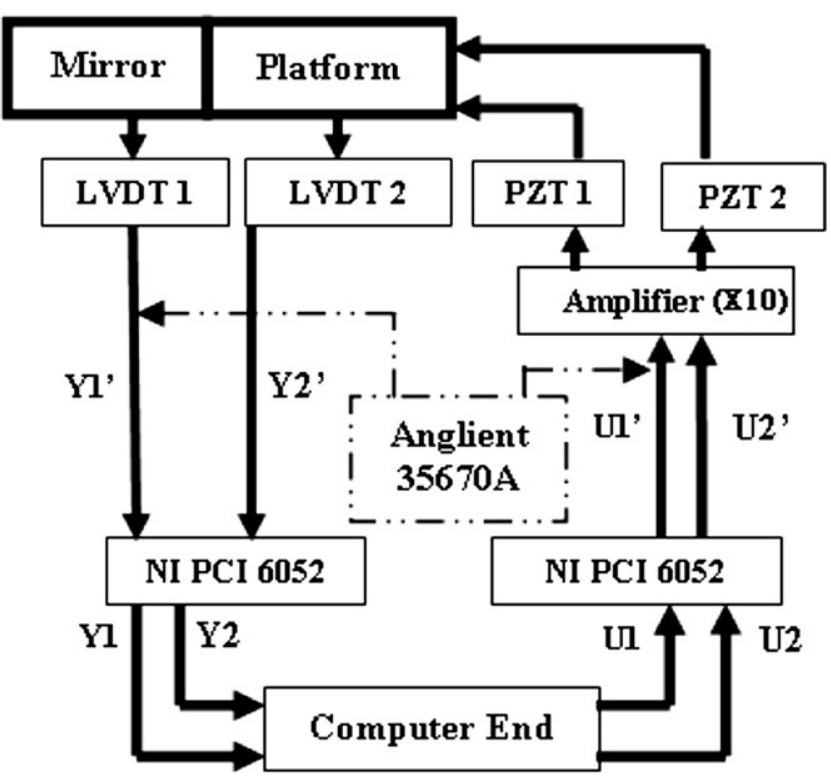

Fig. 3. System control process.

signal analyzer to measure the responses and to carry out data regression. The Agilent 35670A is a SISO instrument. It is also necessary to verify the system linearity by examining the superposition relationships among the transfer functions relating different input-output pairs [9]. Eqs. (1)-(4) lists the system transfer functions where $G_{11}(s)$ stands for the transfer function between input-output pair $U_{1}$ to $Y_{1}$ and so forth. Ref. [9] also presented the comparisons between the simulations and the experimental results of the SISO subsystem and the overall MIMO system to show good agreements.

$G_{11}(s)=\frac{-12.77 s+4.068 \mathrm{e} 4}{s^{2}+1015 s+8.162 \mathrm{e} 6}+\frac{13.43 s-5.316 \mathrm{e} 4}{s^{2}+1171 s+3.869 \mathrm{e} 5}$ 


$$
\begin{aligned}
& G_{21}(s)=\frac{13.19 s-3.568 \mathrm{e} 4}{s^{2}+976.5 s+8.664 \mathrm{e} 6}+\frac{-13.71 s+4.542 \mathrm{e} 4}{s^{2}+1383 s+4.814 \mathrm{e} 5} \\
& G_{12}(s)=\frac{13.52 s-3.303 \mathrm{e} 4}{s^{2}+913.5 s+8.419 \mathrm{e} 6}+\frac{-14.08 s+4.364 \mathrm{e} 4}{s^{2}+1247 s+4.025 \mathrm{e} 5} \\
& G_{22}(s)=\frac{-11.12 s+4.364 \mathrm{e} 4}{s^{2}+964.6 s+8.222 \mathrm{e} 6}+\frac{11.6 s-5.451 \mathrm{e} 4}{s^{2}+1242 s+3.938 \mathrm{e} 5}
\end{aligned}
$$

\section{Theory introduction and system simulation}

This section introduces the MIMO ILC setup and the CILC simulation model. A modified CILC simulation model termed the continuous no-reset switching iterative learning control (CNRSILC) will also be introduced. The CILC simulation model follows the setup in [8], but use the same control criteria as in traditional ILC. As expected, the error response gradually diverges due to the fact that the simulation violated the reset hypothesis. It is observed that the accumulated high frequency vibration at the beginning of the iteration causes the response to go unstable. This observation leads to the idea of the CNRSILC which enables the learning mechanism only after the high frequency oscillation has diminished.

The ILC system is regarded as a two degree-of-freedom system because its control dynamic behavior follows two different guidelines, one is the regular time indices and the other is the iterative runs. The system reacts to the regular time steps, and the iterative run displays the number of system learning iterations. As the number of iteration increases the system response improves gradually.

\subsection{The MIMO ILC algorithm}

The structure of ILC was introduced in [10]. Notice that the previous ILC results in [10] were conducted in the SISO setup. It is necessary to derive the MIMO formulation for this application. We use $t$ to represent the time steps and $k$ to represent the number of iteration. By assuming that the initial condition resets at the start of the iteration, the initial closed-loop control system becomes time invariant. The learning error is defined in Eq. (5) where $Y_{k}(t) \in R^{m \times 1}$ is the system output at the $k$ th iteration and $Y_{d}(t) \in R^{m \times 1}$ is the periodic system reference. The ILC control input is shown in Eq. (6) where $U_{k}(t) \in R^{m \times 1}$ stands for the system input at the $k$ th iteration, $M(q)$ and $L(q)$ can be constant gains or low pass filters for robustness consideration. Eq. (7) is the system output with $T_{\mathrm{c}}(q)$ representing the initial close loop transfer function matrix.

For the two inputs and two outputs system, assume $Y_{d}(t), Y_{k}(t)$, $U_{k}(t), E_{k}(t)$ to be the system references, outputs, ILC inputs, and tracking errors, respectively. The control design is as follows where $M(q)$ and $L(q)$ are diagonal matrices with constant or low pass filter in the diagonal terms.

$$
\begin{aligned}
& E_{k}(t)=Y_{d}(t)-Y_{k}(t) \\
& U_{k+1}(t)=M(q)\left[U_{k}(t)+L(q) E_{k}(t)\right] \\
& Y_{k}(t)=T_{c}(q) Y_{d}(t)+T_{c}(q) U_{k}(t)
\end{aligned}
$$

where $\quad Y_{d}(t)=\left[\begin{array}{l}y_{d 1}(t) \\ y_{d 2}(t)\end{array}\right], \quad Y_{k}(t)=\left[\begin{array}{l}y_{1, k}(t) \\ y_{2, k}(t)\end{array}\right], \quad E_{k}(t)=\left[\begin{array}{l}e_{1, k}(t) \\ e_{2, k}(t)\end{array}\right]$, $U_{k}(t)=\left[\begin{array}{l}u_{1, k}(t) \\ u_{2, k}(t)\end{array}\right], \quad L(q)=\left[\begin{array}{ll}l_{11}(q) & 0 \\ 0 & l_{22}(q)\end{array}\right], \quad C(q)=\left[\begin{array}{ll}c_{11}(q) & c_{12}(q) \\ c_{21}(q) & c_{22}(q)\end{array}\right]$, $G(q)=\left[\begin{array}{ll}g_{11}(q) & g_{12}(q) \\ g_{21}(q) & g_{22}(q)\end{array}\right]$, and $\left.T_{\mathrm{c}}(q)=[I+G(q) C(q)]\right]^{-1} G(q) C(q)$.

By replacing the output term $Y_{k}(t)$ in Eq. (5) with the result from (7) and changing the input term $U_{k}(t)$ into the ILC form shown in (6), one can rebuild Eq. (5) as follows.

$$
\begin{aligned}
E_{k}(t)= & Y_{d}(t)-Y_{k}(t)=Y_{d}(t)-T_{c}(q) Y_{d}(t)-T_{c}(q) M(q)\left[U_{k-1}(t)\right. \\
& -L(q) E_{k-1}(t)
\end{aligned}
$$

Assume $M(q)$ to be the identity matrix, one can derive the error recursion illustrated as Eq. (9).

$$
\begin{aligned}
E_{k}(t) & =Y_{d}(t)-Y_{k-1}(t)-T_{c}(q) L(q) E_{k-1}(t) \\
& =\left[I-T_{c}(q) L(q)\right] E_{k-1}(t)
\end{aligned}
$$

$E_{k}(t)=W^{k}(q) E_{0}(t)$, where $W(q)=\left[I-T_{c}(q) L(q)\right]$

To have asymptotically zero iterative error, it is necessary to assume $M(q)$ to be an unit matrix and recursively replace the iterative item in Eq. (8). Getting asymptotic stable result, all eigenvalues of the matrix $W(q)$ must have modulus less than one. According to this, it is apparent that matrix $W^{k}(q)$ in Eq. (9) approaches zero matrix with $k \rightarrow \infty$ and consequently does the error matrix $E_{k}(t)$. Briefly speaking, one achieves asymptotically zero on the iterative error in the MIMO system as long as the designed ILC inputs gratify the constraint that $M(q)$ is an identity matrix and all eigenvalues of the matrix $W(q)$ have modulus less than one.

Figs. 4 and 5 show the frequency response of $w(q)$ in the SISO case using where the close loop $t_{\mathrm{c}}$ is formed by the subsystem $G_{11}$ and the common PID controller $C_{11}$. Several kinds of learning filter $l(q)$ which was designed at different corner frequency $\left(f_{\mathrm{c}}\right)$ and gain value were selected to check the effects of the learning filter of $w(q)$. Fig. 4 shows the frequency response of $w(q)$, where the learning filter $l(q)$ varied in $f_{\mathrm{c}}$ and $m(q)$ was an unit gain in all tests. The condition on $w(q)$ is satisfied at low frequency. It reveals that the system may converge faster when using the learning filter with higher $f_{\mathrm{c}}$, as long as the signal is within the limited operation frequency. On the contrary, if system signal is out of the limited operation frequency, it may lead the system to diverge easily. Fig. 5 shows the response of $w(q)$ using the filter $l(q)$ with different gains. The $f_{\mathrm{c}}$ of the filter $l(q)$ is $10 \mathrm{~Hz}$ and $m(q)$ is again an unit gain. The results demonstrate that bigger gains may lead to better convergent results, as long as the signal frequency is within the limited operation frequency.

Figs. 6 and 7 are responses of the MIMO ILC constraint, all the eigenvalue modulus of $W(q)$ have to be less than one, when using learning filters $\left(l_{11}=l_{22}\right)$ different in $f_{\mathrm{c}}$ and gain value. Similar to the SISO ILC constraint results, the larger gain leads to better convergent rate in the low frequency region. The most interesting part of the MIMO ILC constraint is that all the frequency responses of the eigenvalue modulus approach one at high frequency. This

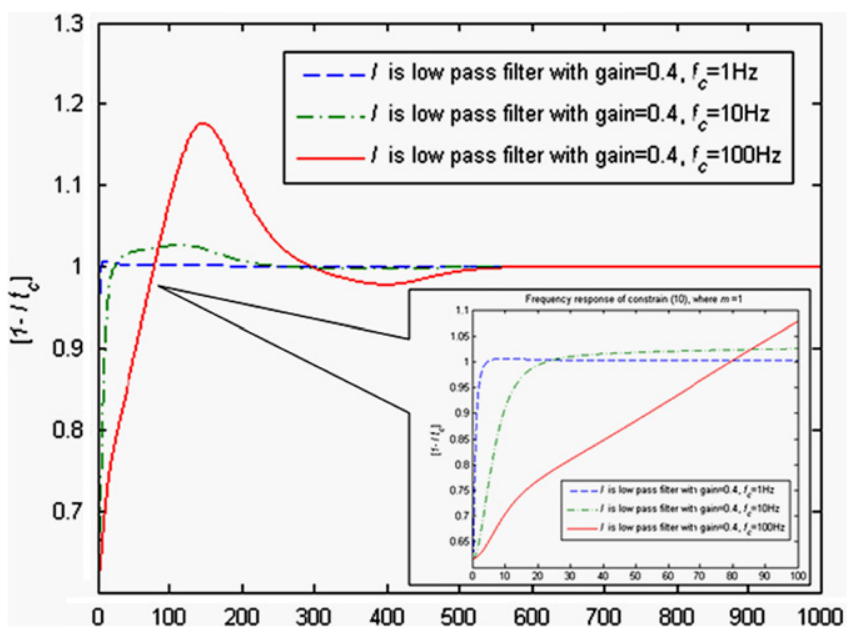

Fig. 4. Frequency response of $w(q)$ where the filter $l(q)$ differs in $f_{\text {c. }}$. 


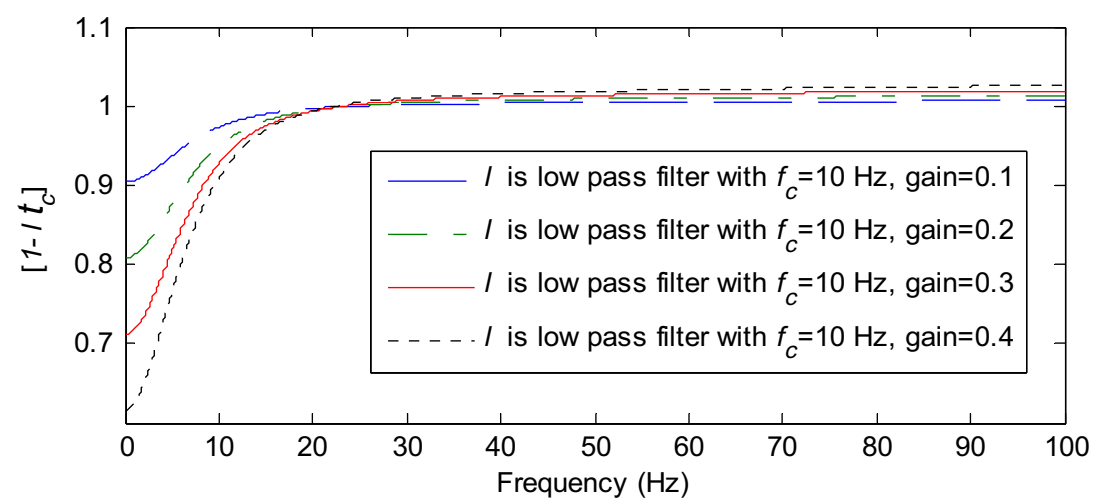

Fig. 5. Frequency response of $w(q)$ where the filter $l(q)$ differs in gain value.

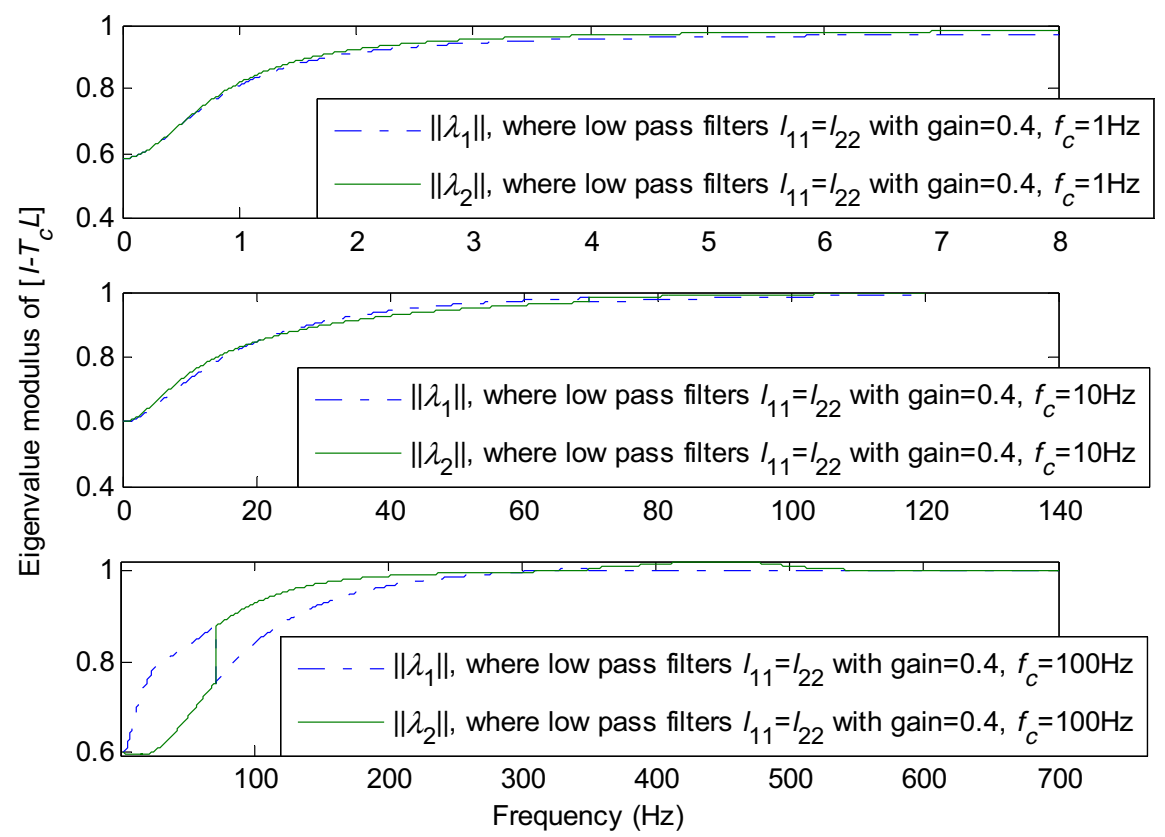

Fig. 6. Frequency response of eigenvalue modulus of the matrix $W(q)$ where the diagonal learning filters $\left(l_{11}=l_{22}\right)$ of $L(q)$ differs in $f_{\text {c }}$.
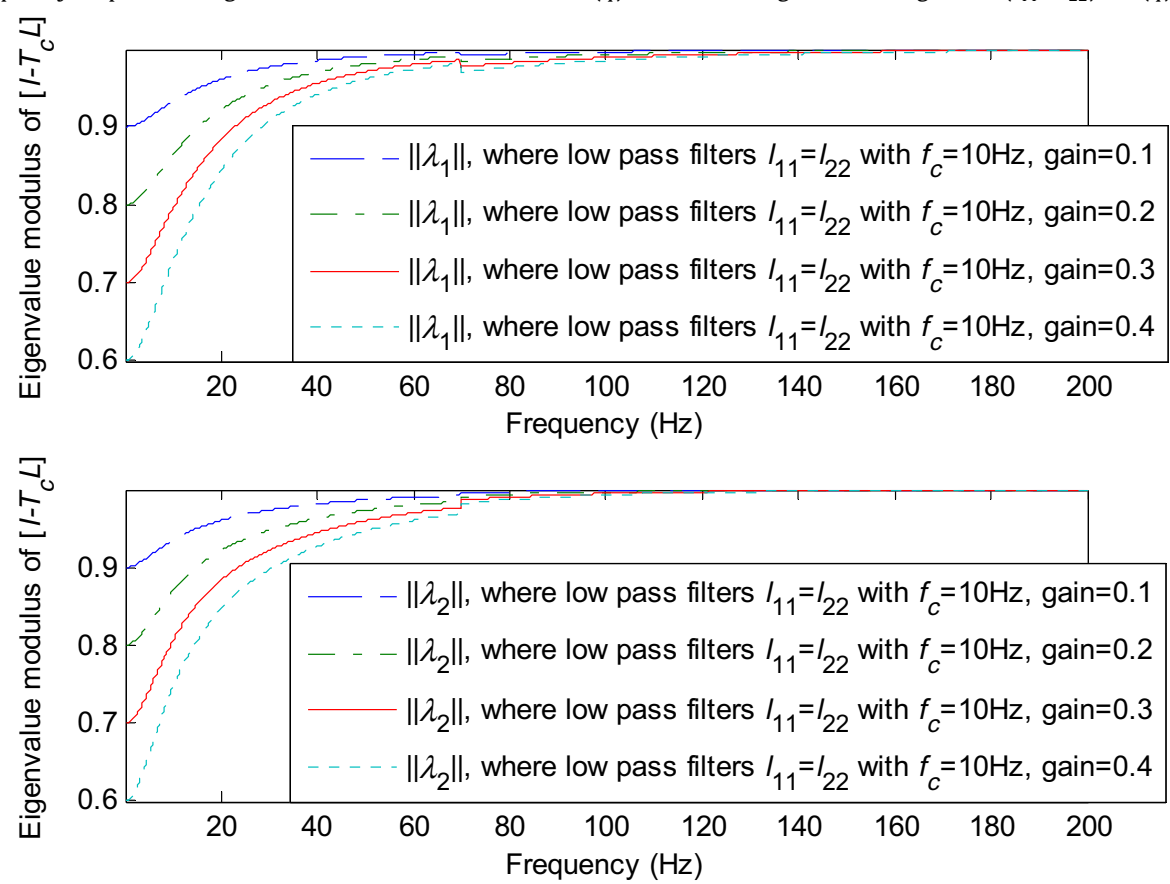

Fig. 7. Frequency response of eigenvalue modulus of the matrix $W(q)$ where the diagonal learning filters $\left(l_{11}=l_{22}\right)$ of $L(q)$ differs in gain. 


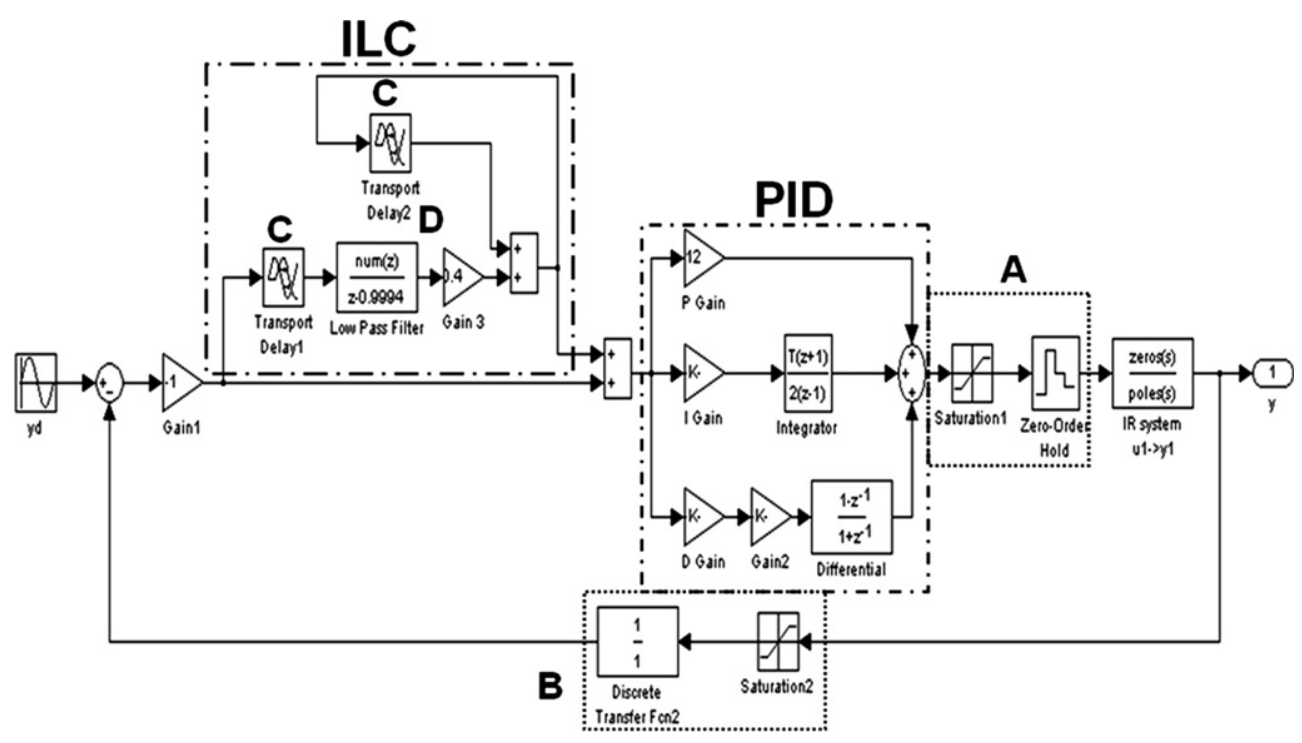

Fig. 8. SISO CILC simulation block diagram.

may be the reason that the error responses at the un-differentiable points do not lead to divergence which is shown in Fig. 15.

\subsection{The control design of the CNRSILC}

As mentioned before, the architecture of the CILC is the same as ILC applied nonstop to the iterative control. Ref. [8] did not explain how to apply the CILC idea, and the so-called stabilizing control design is incomplete. It is believed that the basic principle should still be that the same ILC constraints on $M(q)$ and $L(q)$ should be met. After careful examination over the previous simulation results, we propose to endow the CILC simulation model with a switch control to prevent high frequency oscillation from accumulating.

\subsubsection{The simulation model of the SISO CILC}

The SISO CILC simulation model using subsystem transfer function $G_{11}$ and PID control $C_{11}$ to build the closed-loop transfer function $t_{c}$ is shown in Fig. 8, where the blocks " $A$ ", " $B$ " are used to model $\mathrm{A} / \mathrm{D}$ and $\mathrm{D} / \mathrm{A}$ functions. The part encircled by dashed lines is the ILC controller including a periodic iterative delay block $C$ and a low pass filter block $D$. The simulation used a $1 \mathrm{~Hz}$ sine wave as the reference signal and neglected the initial state error prob-
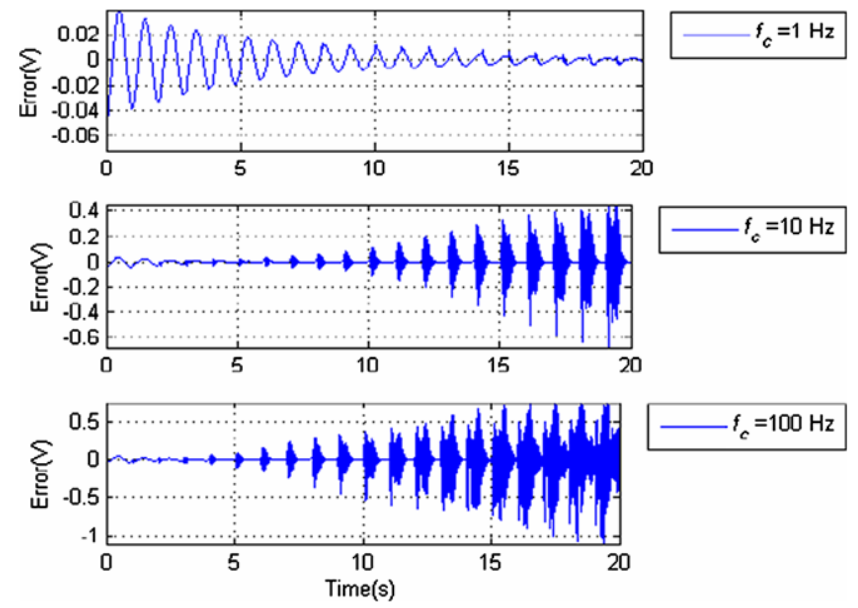

Fig. 9. SISO CILC error response where $m(q)=1$ and the learning filter $l(q)$ varies in $f_{\mathrm{c}}($ Gains $=0.4)$. lems. The simulation then allowed the iterative signals to run nonstop feedback to the system. The iterative error responses are illustrated in Fig. 9. It can be observed that the error converging situations basically followed the predicted results in Figs. 4 and 5, the smaller $f_{\mathrm{c}}$ of the learning filter $l(q)$ resulted in slower convergence speed. Unfortunately gradually increasing high frequency oscillations were induced at the beginning of each new iteration cycle after the tracking errors had approached zero disregard the efforts for tuning filter $l(q)$. The high frequency oscillation grow worse when higher $f_{\mathrm{c}}$ is used for the filter $l(q)$. It is also observed that larger $l(q)$ gains lead to faster convergent rates as shown in Fig. 10, together with a diverging high frequency oscillation at the beginning of each iteration cycle. From the above simulations, it is apparent that continuously running ILC without reset irrespective to any modification is similar to using ILC with unbounded initial state errors.

\subsubsection{The simulation model of the SISO CNRSILC}

The practical application of continuous no-reset ILC does not allow additional constraints to the calculated or the measured error at the beginning of each iteration cycle. After through examination,
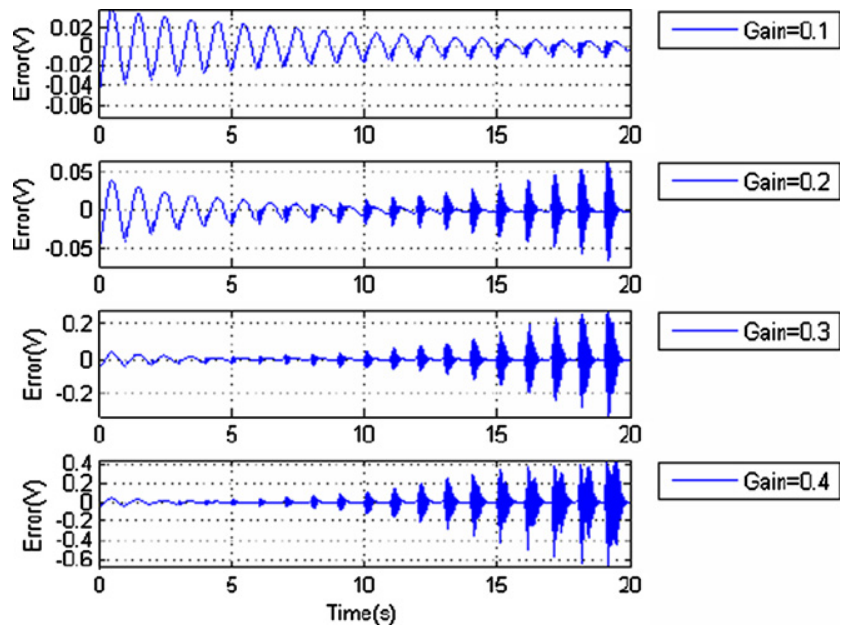

Fig. 10. SISO CILC error response where $m(q)=1$ and the learning filter $l(q)$ varies in gains $\left(f_{\mathrm{c}}=10 \mathrm{~Hz}\right)$. 


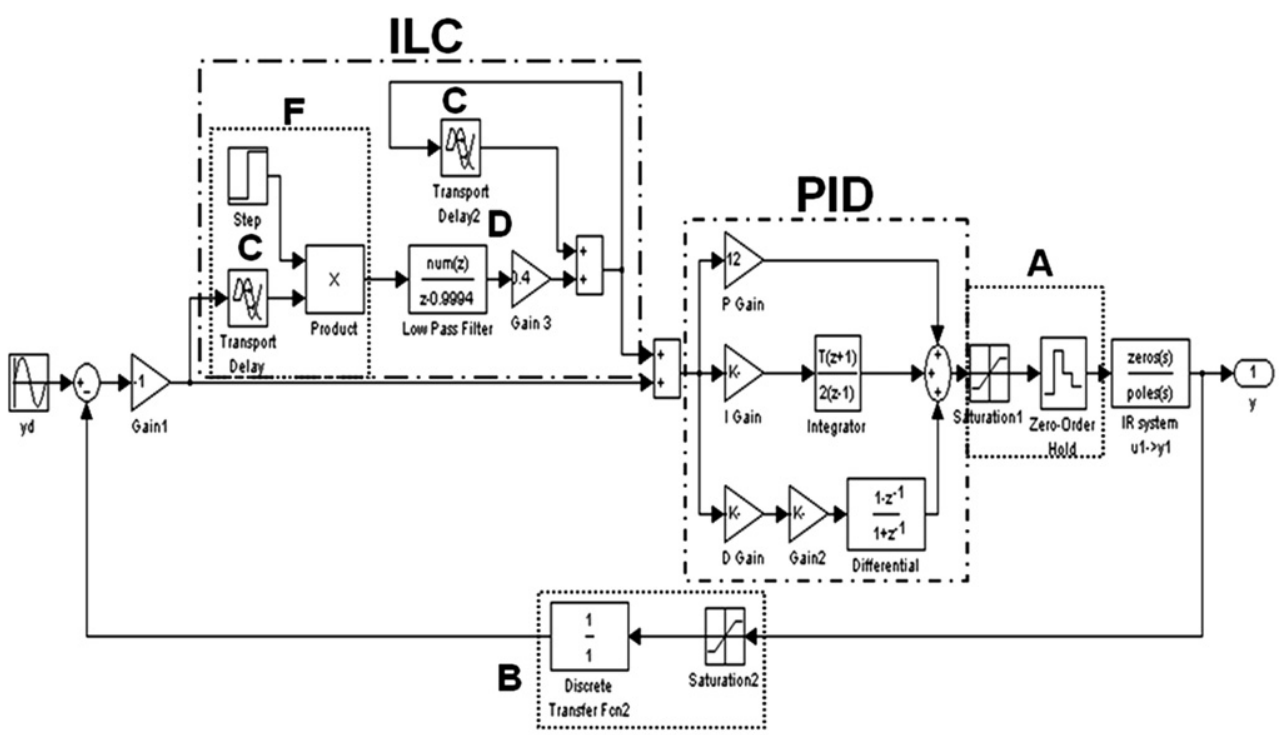

Fig. 11. SISO CNRSILC simulation block diagram.

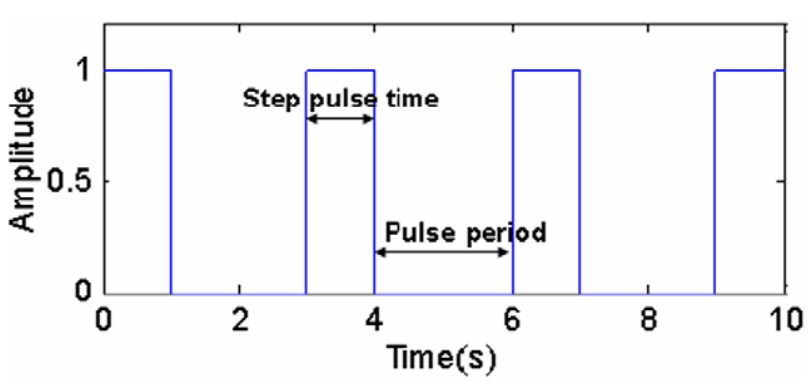

Fig. 12. The pulse signal of the switch block $F$.

it occurred to the authors that the iterative learning algorithm stores the oscillation from the last iteration and injects it back into the next iteration cycle. This accumulated oscillation eventually prevails and the ILC would diverge. Because the initial error accumulates, a reasonable approach would be to enable the learning mechanism only when needed. This is easily implemented by inserting a switch to the ILC controller and closes the switch only periodically. Fig. 11 is the CNRSILC simulation block diagram. There is an additional pulse switch block $F$ to control the error accumu-
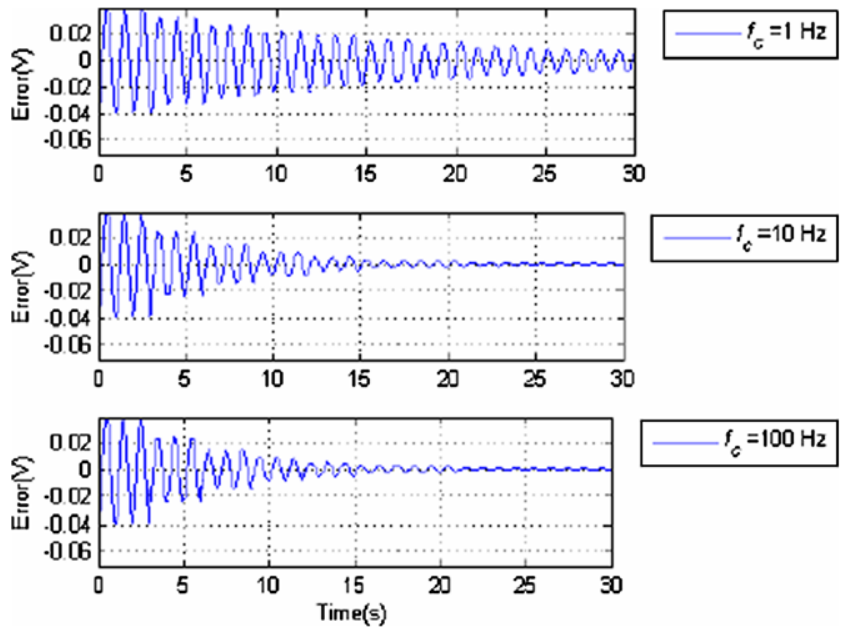

$f_{c}=100 \mathrm{~Hz}$

Fig. 13. SISO CNRSILC error response where $m=1$ and the learning filter $l(q)$ varies in $f_{\mathrm{c}}($ Gains $=0.4)$.
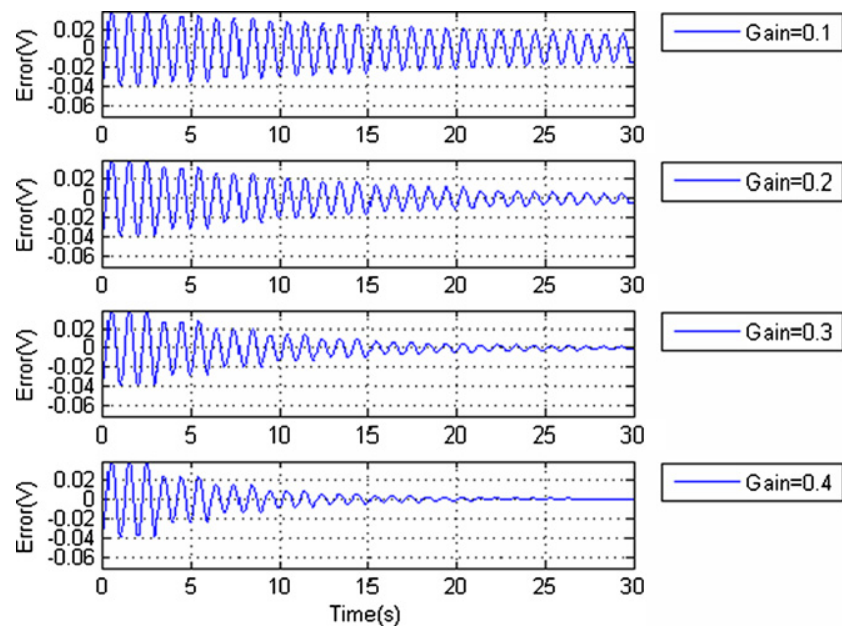

Fig. 14. SISO CNRSILC error response where $m=1$ and the learning filter $l(q)$ varies in gains $\left(f_{\mathrm{c}}=10 \mathrm{~Hz}\right)$.

lation, and Fig. 12 is the pulse switching signal of the switch block $F$. It should be noted that the pulse period should be more than twice the integer multiple of the reference period, and that the pulse duration should be equal to the reference period. The simulation results of a $1 \mathrm{~Hz}$ sine wave following are shown in Figs. 13 and 14 , where $m(q)$ is an unit gain and $l(q)$ is a low pass filter with different $f_{\mathrm{c}}$ and gain values. By observing these tracking error responses, CNRSILC not only suppresses the tracking error but also avoids the high frequency oscillation. There is a good indication that the proposed CNRSILC method is practical from the simulation point of view. The results shown in Fig. 13 have also verified the frequency response of the constraint on $W(q)$. The learning filter with a higher $f_{\mathrm{c}}$ still leads to better convergent rate, and subsequently, the error response in Fig. 14 also confirms that the learning filter with a larger gain results in faster convergence. The study also tested different pulse period to understand the effect of the switching function. The convergence rate of the equivalent iteration number is exactly the same, no matter what pulse period was chosen. These simulations confirms that adding a switch to ILC controller can suppress the reset demand and achieve asymptotic stable response without any restriction on the initial state for the SISO case. 

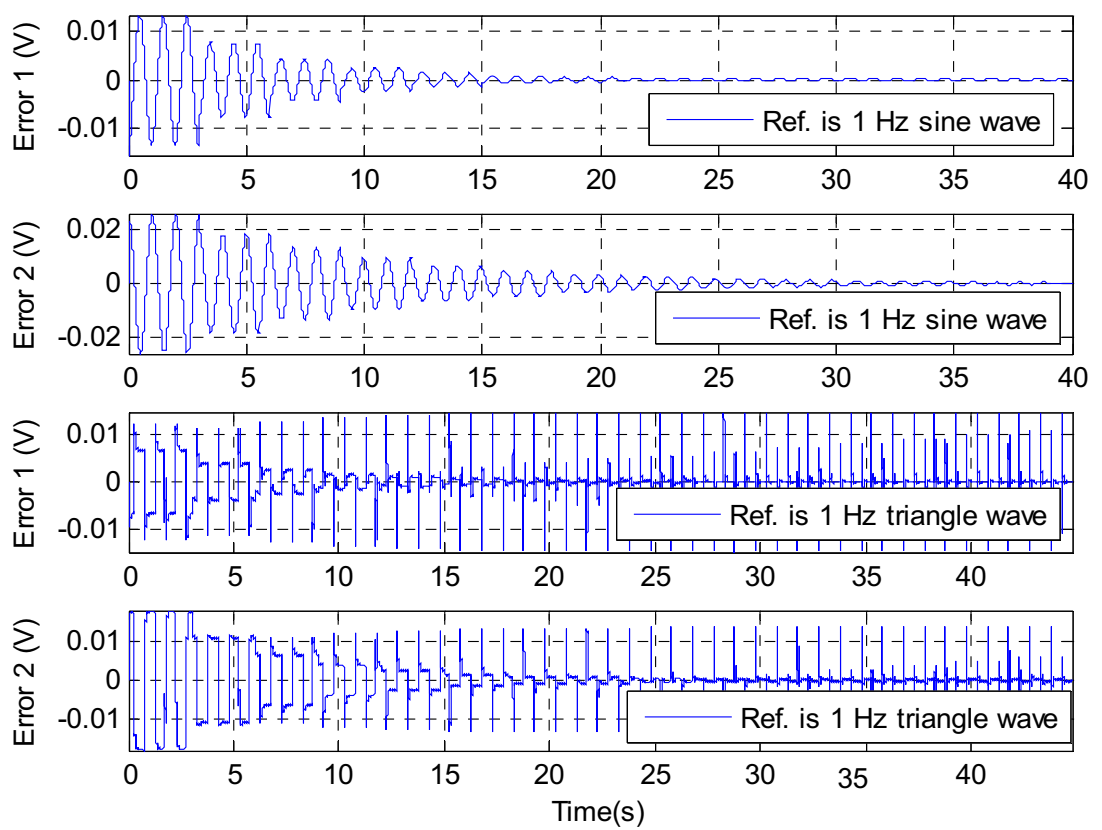

Fig. 15. MIMO CNRSILC Error response, where $M$ is an unit matrix and the diagonal filters $l_{11}=l_{22}$ of $L(q)$ are low pass filters with $f_{\mathrm{c}}=10 \mathrm{~Hz}$ and gain $=0.4$.
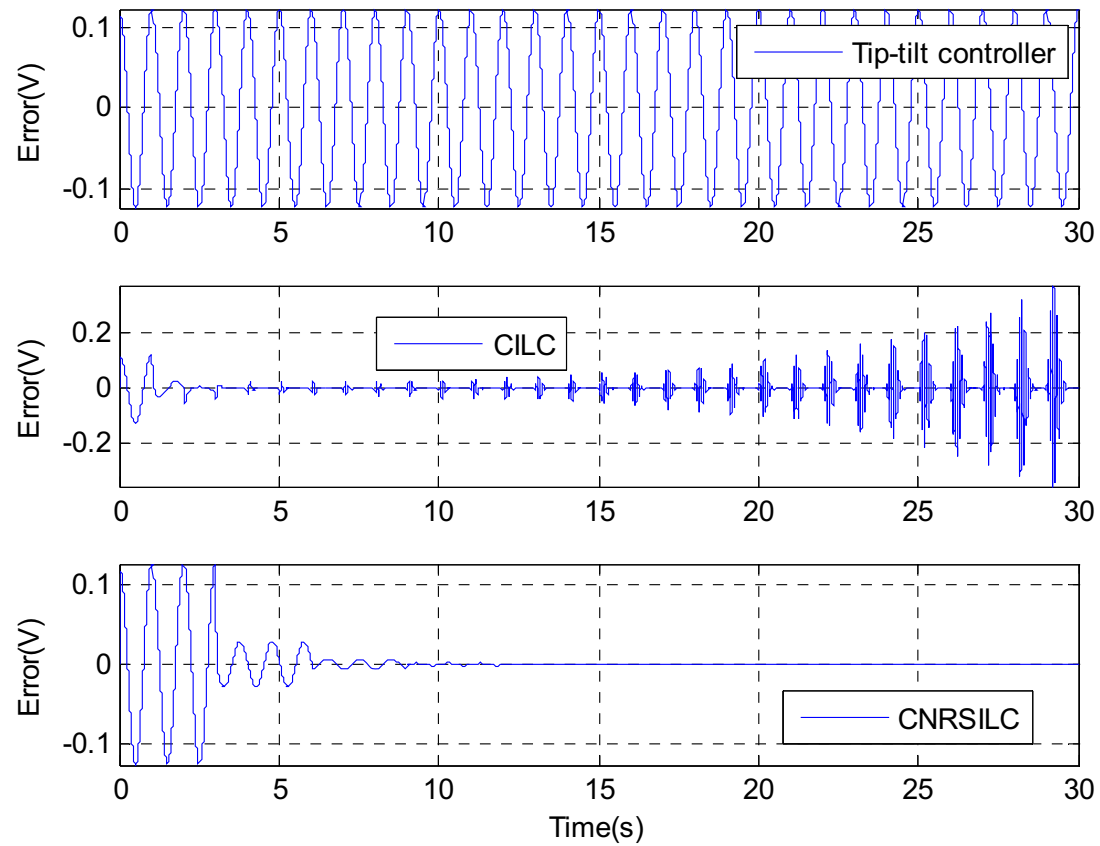

Fig. 16. Simulation error response compare plot for the tip-tilt mirror [11]

\subsubsection{The simulation model of the MIMO CNRSILC}

Fig. 15 shows the MIMO CNRILC simulation error response of the two-input-two-output test system using two different references, a reversed sine wave and a triangular wave. These results again confirm that the CNRSILC method achieves excellent control efforts but regrettably its performance at the non-differentiable points is poor.

\subsection{The verifications of the CNRSILC method from other servo mechanisms}

To illustrate the control effect, two different servo systems are discussed in the next paragraphs to validate the CNRSILC perfor- mance. The first is the tip-tilt compensator in an adaptive optics system. The function of the tip-tilt mirror in [11] is similar to the mechanism mentioned in Section 2. The second example is the optical disk drives. Optical disk drivers were generally installed in DVD-ROM and CD-ROM, and therefore we present three examples learned from [13-15] to manifest the converging error responses of CNRSILC. All the optical disk systems were controlled by lead-lag controller (LLC) originally.

\subsubsection{The tip-tilt mirror model}

The tip-tilt mirror was modeled by continuous time transfer function $G_{\mathrm{TT}}(s)$ in [11]. Eq. (11) is the simplest transfer function $K(z)$ of the compensator used to gather the closed-loop data. 
(a). Tracking error of DVD
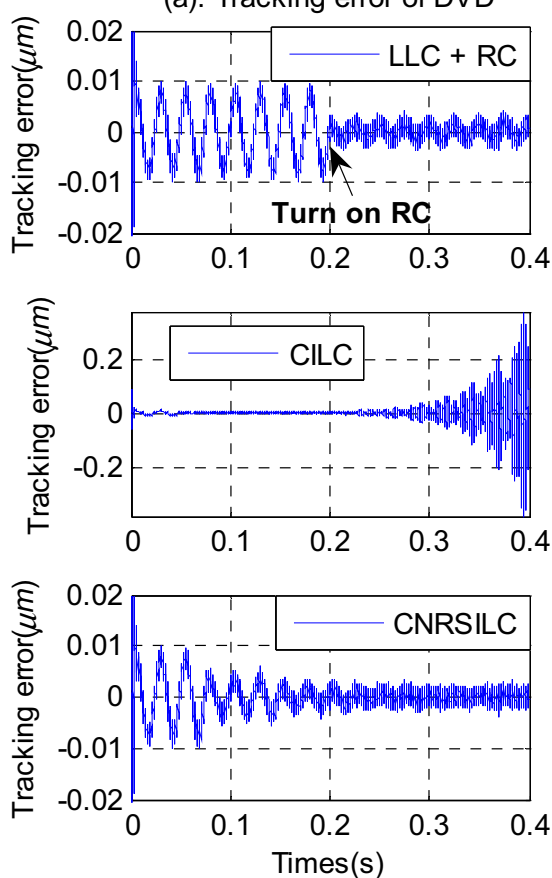

(b). Tracking error of $\mathrm{CD}-\mathrm{ROM}$
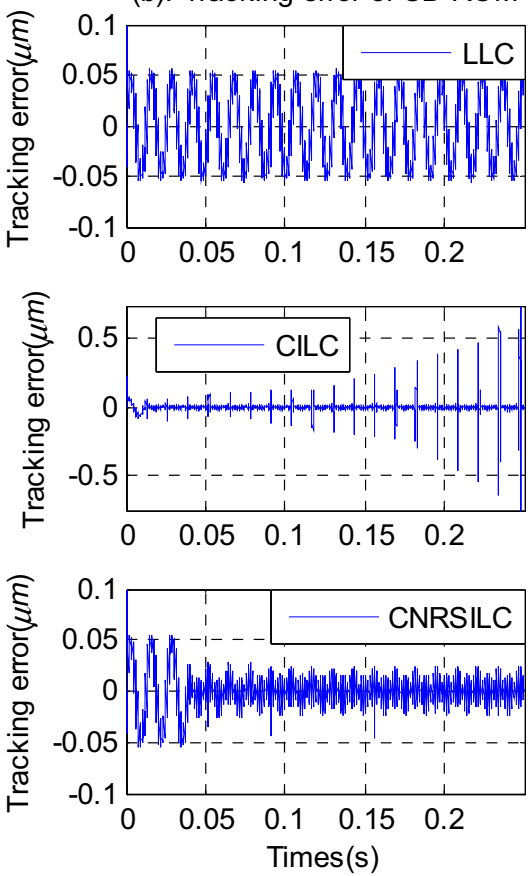

Fig. 17. Simulated error response of optical disk drives [13,14].
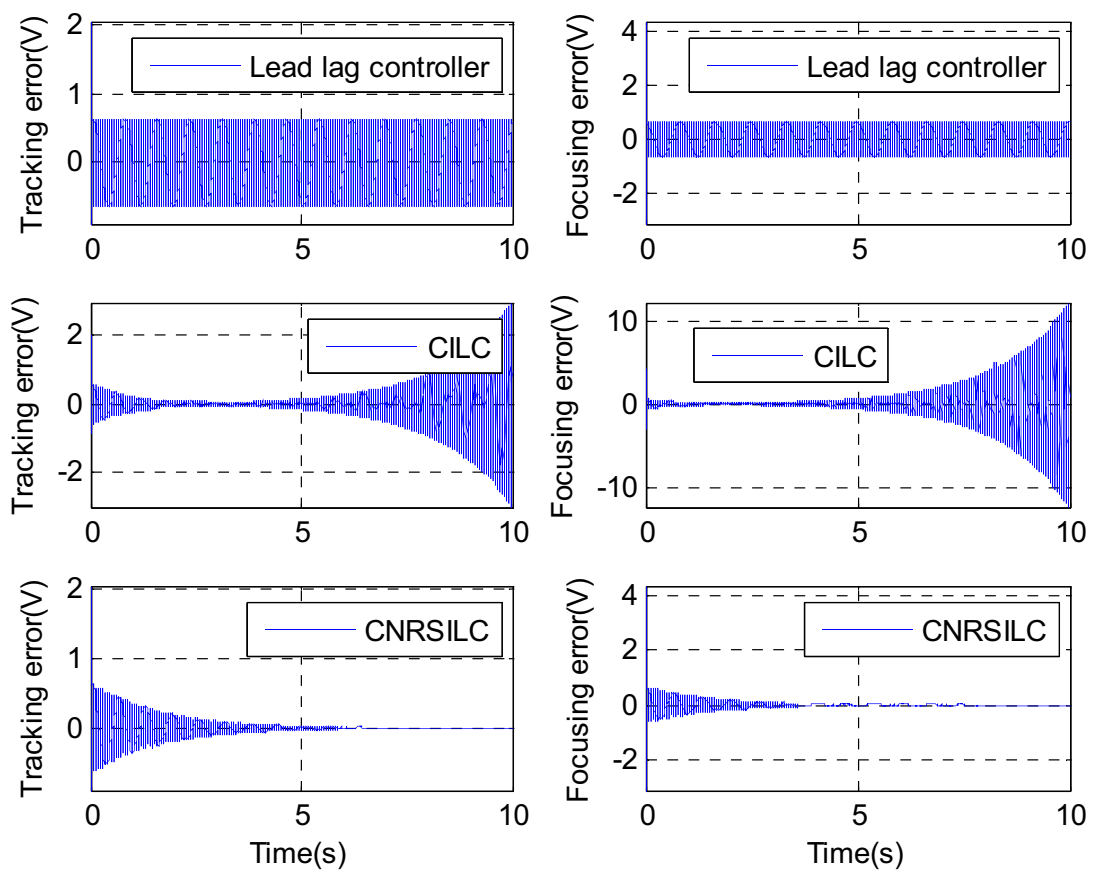

Fig. 18. Simulated error response of optical disk drives of MIMO CD-ROM [15].

$G_{\mathrm{TT}}(s)=\frac{8.52 \times 10^{10}}{s^{3}+1.32 \times 10^{4} s^{2}+5.808 \times 10^{7} s+8.52 \times 10^{10}}$

$K(z)=\frac{0.05 z}{z-1}$

It is important to achieve high performance servo algorithm for the tip-tilt (TT) loop of the adaptive optics (AO). The AO system [12] re- quires the use of a natural guide star to elicit tip and tilt information which is not available from the laser target. Fig. 16 is the comparison of the simulation results with the original controller, the CILC and the CNRSILC. Apparently, the CNRSILC works best.

\subsubsection{The optical disk model}

The tracking actuator model of the DVD-ROM is learned from [13]. In [13], an add-on controller, repetitive controller (RC), was 

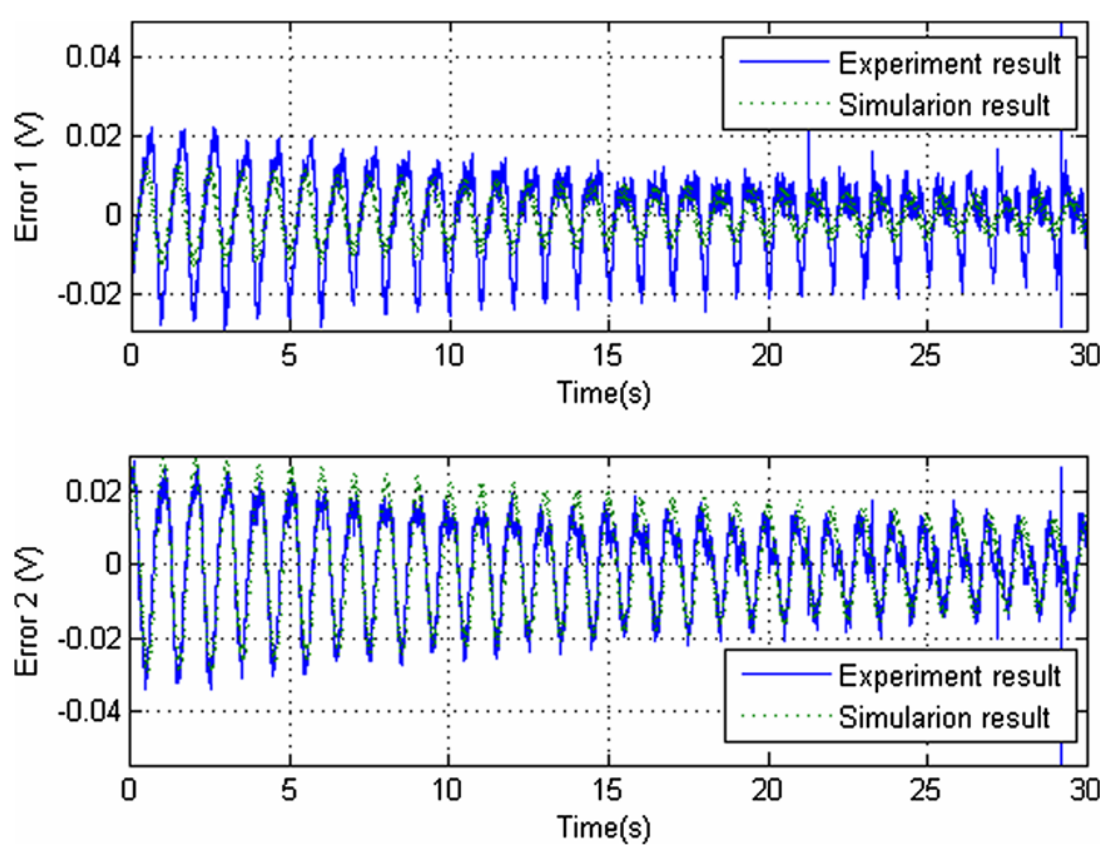

Fig. 19. [Exp. 1] MIMO CNRSILC error response where $M$ is an unit matrix and the diagonal filters $l_{11}=l_{22}$ of $L(q)$ are low pass filters $\left(f_{\mathrm{c}}=10 \mathrm{~Hz}\right.$ and gain $\left.=0.1\right)$.
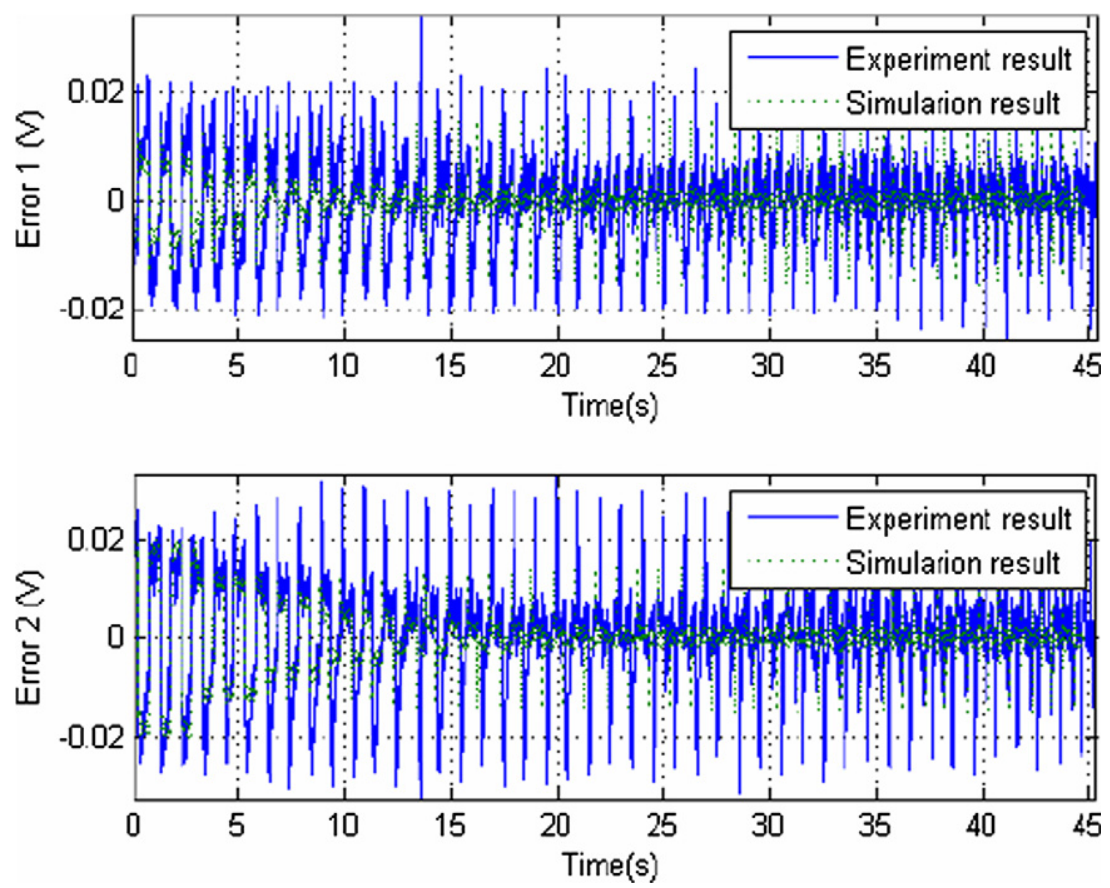

Fig. 20. [Exp. 2] MIMO CNRSILC error response where $M$ is an unit matrix and diagonal filters $l_{l l}=l_{22}$ of $L(q)$ are low pass filters $\left(f_{\mathrm{c}}=10 \mathrm{~Hz}\right.$ and gain $\left.=0.4\right)$.

used to attenuate the inherent periodic disturbance caused by the inevitable eccentric rotation of disk. The control system of RC in [13] was reconstructed in simulation to exhibit the outstanding controllability again. Also, CILC and CNRSILC were tested in simulation to assess its performance. The frequency of the periodic disturbance was $40 \mathrm{~Hz}$ with white noise when the DVD-ROM rotated at a constant angular velocity of $2400 \mathrm{rpm}$. Fig. 17a shows the simulated tracking error of RC, CILC and CNRSILC in which RC was tuned on at $0.2 \mathrm{~s}$. It is obvious that CNRSILC has asymptotic error and the convergent error of CNRSILC is smaller than RC.
Fig. $17 \mathrm{~b}$ is the simulated error response of optical disk driving system in the 52X CD-ROM. The model which consists of the VCM driver, the tracking actuator and the RF-amp was studied from [14]. The control purpose of [14] was to design an anti-shock controller to lessen the external disturbance when the disk rotational frequency set at $77 \mathrm{~Hz}$. Accordingly, the external disturbance trajectory was set to be $15 \sin 483.8 t$ in simulation.

The last model was illustrated in [15] and it was identified from the commercially available $36 \mathrm{X}$ CD-ROM drive. The identified systems for fine actuator system and focusing actuator system were 

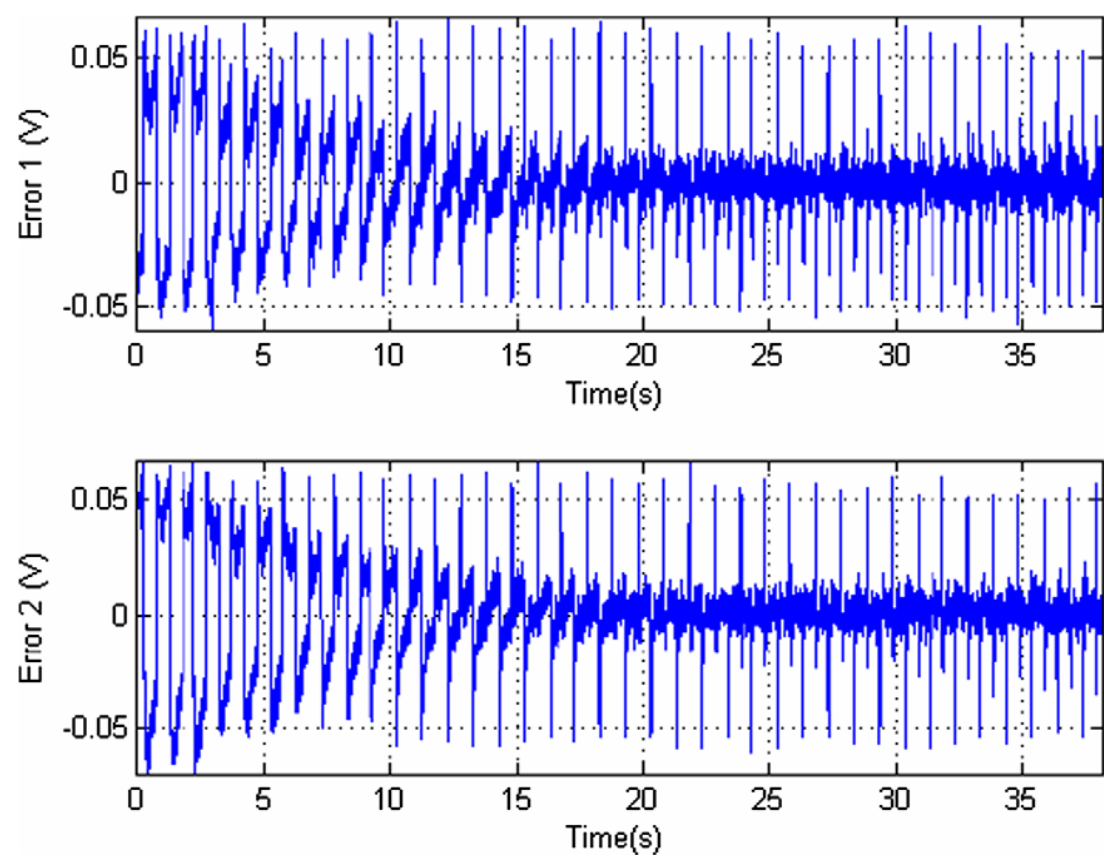

Fig. 21. [Exp. 3] MIMO CNRSILC error response where Ref. is $1 \mathrm{~Hz} 512$ steps triangle wave, $M$ is an unit matrix and the diagonal filters $l_{11}=l_{22}$ of $L(q)$ are low pass filters. $\left(f_{\mathrm{c}}=10 \mathrm{~Hz}\right.$ and gain $\left.=0.4\right)$.
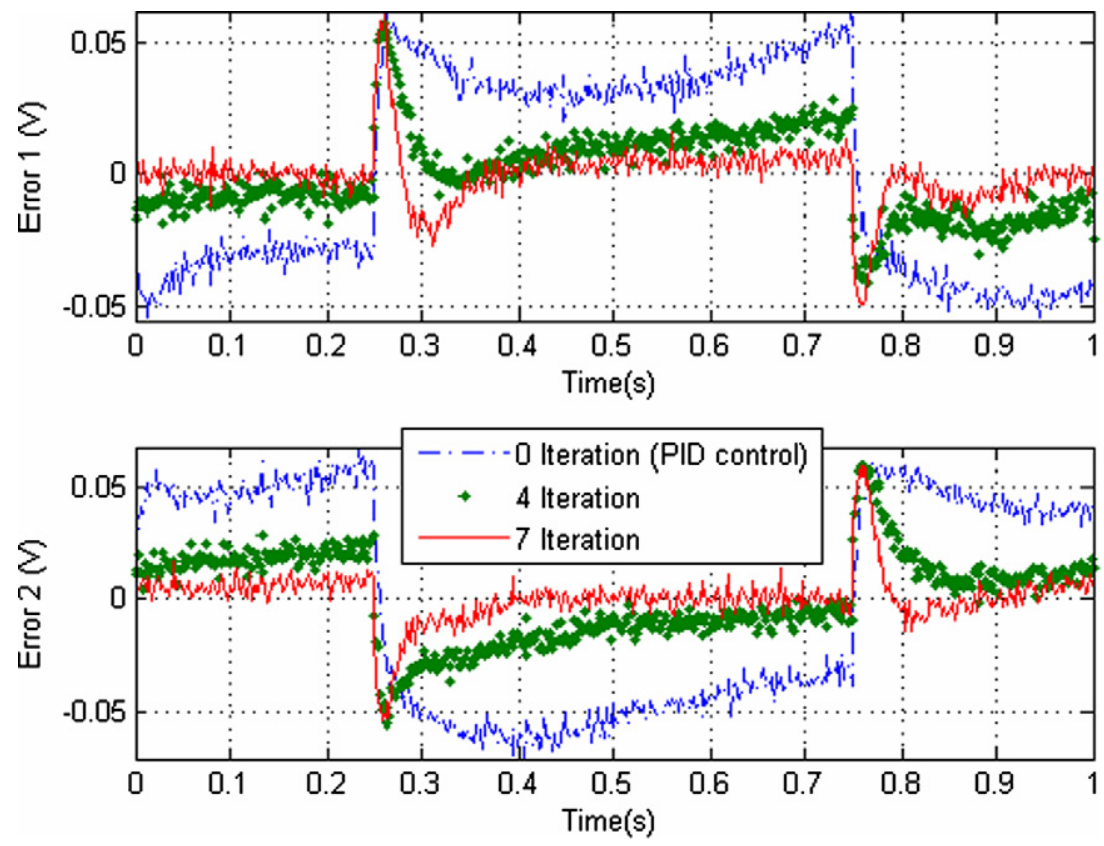

Fig. 22. [Exp. 3] Iteration error comparison plot from Fig. 20.

viewed as the nominal models respectively. In order to simplify the system and considering the main coupling effect of the optical disk drive, the input item $\mathrm{h}$ which was the distance between $O_{\mathrm{c}}$ (the mass center of the lens holder) and the beam spot was neglected, and the system was then regarded as a two by two plant. The reference trajectory of the simulation was [100sin 138.23t $100 \sin 138.23 t]^{\mathrm{T}}$. The MIMO CNRSILC error responses for the fine tracking and focusing servo are shown in Fig. 18. It is clear that MIMO CNRSILC also achieved better performance. Above simulated error responses all demonstrate that CNRSILC can improve error performance and fulfill perfect tracking iteration-by-iteration.

\section{Experiment results}

The simulation results indicated that the CNRSILC method is effective in the no-reset application of the ILC problem. The experimental study involved a setup as shown in Fig. 3. There were three types of reference command signals tested: opposite sine waves, triangular waves, and 512 step-scan triangular waves. The reason for using sine waves, triangle waves was to enable the comparison between the experimental and simulation results. The 512 stepscan triangle wave reference came from the specification of the standard high resolution step-and-scan operation. The reference 
scanning frequency and the sampling rate for the following experiments were set to $1 \mathrm{~Hz}$ and $10 \mathrm{KHz}$. The experiments compared error responses from common PID control with the CNRSILC to confirm a superior convergent tracking performance.

\subsection{Experiment 1: using $1 \mathrm{~Hz}$ sine wave references}

It is clear that the error performances of CNRSILC method were similar in both the experiments and the simulations from Fig. 19. The tracking errors converged gradually with the iteration. This experiment proved that the proposed CNRSILC method demonstrated good performance not only in the simulation but also in practical applications. The following experiment confirmed the triangle waves operation to accomplish constant speed scanning. The experiment results were also very similar to the simulation results.

\subsection{Experiment 2: using $1 \mathrm{~Hz}$ triangle wave references}

Fig. 20 showed the non-converging and non-diverging high frequency oscillation for the triangular references at the turning points where scanning direction changed. These results also matched the simulation results.

\subsection{Experiment 3: using $1 \mathrm{~Hz} 512$ step-and-scan triangle wave references}

The 512 step-and-scan triangular wave test uniformly divided the triangular wave into 512 steps. The frequency of the wave was $1 \mathrm{~Hz}$ so that the stopping time of each step was $1 / 512 \mathrm{~s}$, in other words, each step was constituted of 20 sampling points only. The traditional PID control in this case experienced extremely insufficient time for the response to settle. Using CNRSILC, however, could clearly improve the settling time and allow the response to settle well within the stepping duration as shown in Fig. 22. Although, there were still non-converging and non-diverging peak errors at the turning points shown in Fig. 21.

\section{Conclusion}

This paper proposed a CNRSILC algorithm for a high performance novel optical scanner. The system represented a typical ILC application and suffered the common ILC problem of diverging initial error. This paper first presented an MIMO version of the ILC control constraints, and used computer simulations to verify the analysis. The simulation also demonstrated the effects of accumulated initial error. The paper then moved on to propose the contin- uous no-reset switching iterative learning control algorithm. Although a rigorous stability analysis is not yet complete, the application of CNRSILC on several other servo mechanisms all proved that the method was effective in relaxing the reset assumption on the initial conditions. Both the simulation results and the experimental results confirmed the performance of the proposed control. The experiment on the step-and-scan tests further demonstrated the advantage of using the CNRSILC.

\section{Acknowledgements}

This work is supported by the National Science Council, Taiwan under Contract No. NSC 93-2623-7-002-006, and in part by ChungShan Institute of Science and Technology, Materials and ElectroOptics Research Division.

\section{References}

[1] Yeh Y, Yen J, Lee C. A novel high-speed optical scanning platform. Proc SPIE 2005;5638:352-61. EI.

[2] Lee J, Lee H, Bien Z. Iterative learning control with feedback using fourier series with application to robot trajectory tracking. Robotica 1993;11:291-8.

[3] Arimoto S, Kawamura S, Miyazaki F. Bettering operation of robots by learning. J Robotic Syst 1984;1(2):123-40.

[4] Heinainger G, Fenwick D, Paden B, Miyazaki F. Stability of iterative learning control with disturbances and uncertain initial conditions. IEEE Trans Automatic Control 1992;37:110-4.

[5] Lee H, Bien Z. Study on robustness of iterative learning control with non-zero initial error. INT J Control 1996;64(3):345-59.

[6] Sun M, Wang D. Initial shaft issues on discrete-time iterative learning control with system relative degree. IEEE Trans Automatic Control 2003;48(1): 44-148.

[7] Park K. An average operator-based PD-type iterative learning control for variable initial state error. IEEE Trans Automatic Control 2005;50(6):865-9.

[8] Sison LG, Chong EKP. No-reset iterative learning control. In: Proceedings of the 35th conference on decision and control, Kobe, Japan; 1996.

[9] Yeh Y, Yen J, Peng Y, Lee J. High performance motion control of the novel optical servo scanning platform. Proc SPIE 2005;5873:183-92. EI.

[10] Norrlöf M. Iterative learning control: analysis, design and experiments. PhD Thesis No. 653. Department of Electrical Engineering, Linköping University; 2000. ISSN 0345-7524.

[11] Looze DP, Dam MA, Johansson EM. Comparison of tip-tilt controllers using the STRAP wavefront sensor in the W.M. Keek observatory laser guide start adaptive optical system. Proc SPIE 2006;6272.

[12] Wizinowixh PL et al. The W.M. Keck laser guide star adaptive optics system: overview. Publ Astron Soc Pacific 2006;118:297-309.

[13] Doh T, Ryoo J, Chung M. Design of a repetitive controller: an application to the track-following servo system of optical disk drives. IEE Proc Control Theory Appl 2006;153(3):323-30.

[14] Baek J, Chung C, Tomizuka M. Anti-shock controller design for optical disk drive systems with a nonlinear controller. In: Proceedings of the 2006 American control conference; 2006. p. 1982-9.

[15] Yeh T, Chuan Y. Modeling and identification of opto-mechanical coupling and backlash nonlinear in optical disk drivers. Consumer Electron IEEE Trans 2000;46:105-15. 